ISSN 1991-8631

Original Paper

http://indexmedicus.afro.who.int

\title{
Production du coton et adoption de la culture attelée dans la Commune de Malanville au nord du Bénin
}

\author{
Moussa GIBIGAYE ${ }^{1 *}$, Yves Antoine TOHOZIN $^{1}$ et Yacouba SANON $^{2}$ \\ ${ }^{l}$ Département de Géographie et Aménagement du Territoire /FLASH/UAC, Benin. \\ ${ }^{2}$ Département de Sociologie et d'Economie Rurale/Université de Ouagadougou, Burkina Faso. \\ *Auteur correspondant, E-mail : Moussa_gibigaye@yahoo.fr
}

\section{RESUME}

Une étude par enquête de type micro-économique et de type agronomique auprès des paysans et par observations sur les champs a été conduite dans la Commune de Malanville pour comprendre les facteurs qui influencent l'adoption de la culture attelée. Les résultats montrent que la culture attelée est l'un des facteurs de développement et de modernisation de l'agriculture dans la zone d'étude. Dans cette zone, l'adoption de la culture attelée comme moyen de modernisation de l'agriculture dépend de certaines conditions favorables ou non : i) la disponibilité des bœufs de trait dont les prix varient de $+12 \%$ en moyenne par an ;ii) la disponibilité des équipements agricoles (charrue et charrettes) qui connaissent des inflations d'une campagne à une autre ; et iii) les compétences qui constituent toujours des facteurs de blocage dans ce processus. Ces conditions sont actuellement abîmées par la chute de la production du coton, premier pourvoyeur des revenus. Est-ce le retour à la case de départ qui est annoncé ? Des mesures doivent être prises pour accompagner les producteurs dans la modernisation de l'agriculture sous forme de plan Marshall.

(c) 2012 International Formulae Group. All rights reserved.

Mots clés: Culture attelée, équipements agricoles, bœufs de trait, modernisation de l'agriculture.

\section{INTRODUCTION}

Le financement de l'agriculture en Afrique au Sud du Sahara a été une préoccupation des chercheurs et des acteurs de développement à la base. Introduite pour développer les cultures de rente (Charrière, 1984) et comme moyen de l'association agriculture-élévage (Roupsard, 1894), la culture attelée a été l'un des axes forts des projets de développement qui se sont succédés en Afrique de l'Ouest et du Centre entre 1970 et 1990, tels que décrits par Roesch (2004), et Pingali et al. (1987). La place de la traction animale dans les exploitations agricoles des savanes subsahariennes varie selon les zones agro-écologiques et les systèmes de culture (Vall et al., 2003). La traction animale est adoptée à bien des égards : culture attelée, transport, synergies agriculture-élevage et, les impacts économiques sur la productivité du travail. Cette forme de mécanisation agricole engendre l'augmentation des revenus et des gains de temps dans les exploitations (Barro et al., 2005; Gibigaye et al., 2006 ; Faure, 1994). Mieux, les revenus des cultures industrielles relayés par les opérations de crédit et de vulgarisation ont permis à plusieurs générations de paysans d'adopter la technique (Lhoste, 1995 ; Le Thiec, 1996). Au Bénin en général, et dans les départements du Borgou et de l'Alibori en particulier, l'agriculture, 
notamment la culture du coton, a connu une évolution remarquable grâce à la culture attelée (Gibigaye, 2008). Mais, l'adoption de cette technique aujourd'hui ne cesse de susciter des débats face : i) à la montée des prix des équipements et de leur disponibilité ; ii) aux problèmes de trésorerie importants chez les utilisateurs qui sont en situation d'absence d'une culture de rente (notamment le coton qui connaît des difficultés actuellement au Bénin), élément déterminant de la rentabilité de la traction animale et enfin, iii) aux compétences nécessaires qui doivent accompagner ces technologies.

Cette étude se propose d'analyser les éléments d'appréciation de l'adoption de la culture attelée dans la Commune de
Malanville, notamment le prix des éléments de l'attelage, les facteurs qui limitent l'adoption de cette technique agricole introduite avec l'avènement de la culture du coton.

\section{MATÉRIEL ET MÉTHODES \\ Zone d'étude}

La Commune de Malanville est située à la limite Nord du département de l'Alibori (Figure 1). Elle est limitée au Nord par la République du Niger, au Sud par les Communes de Kandi et de Ségbana, à l'Ouest par la Commune de Karimama et à l'Est par la République Fédérale du Nigeria. Elle couvre une superficie de $3016 \mathrm{~km}^{2}$.

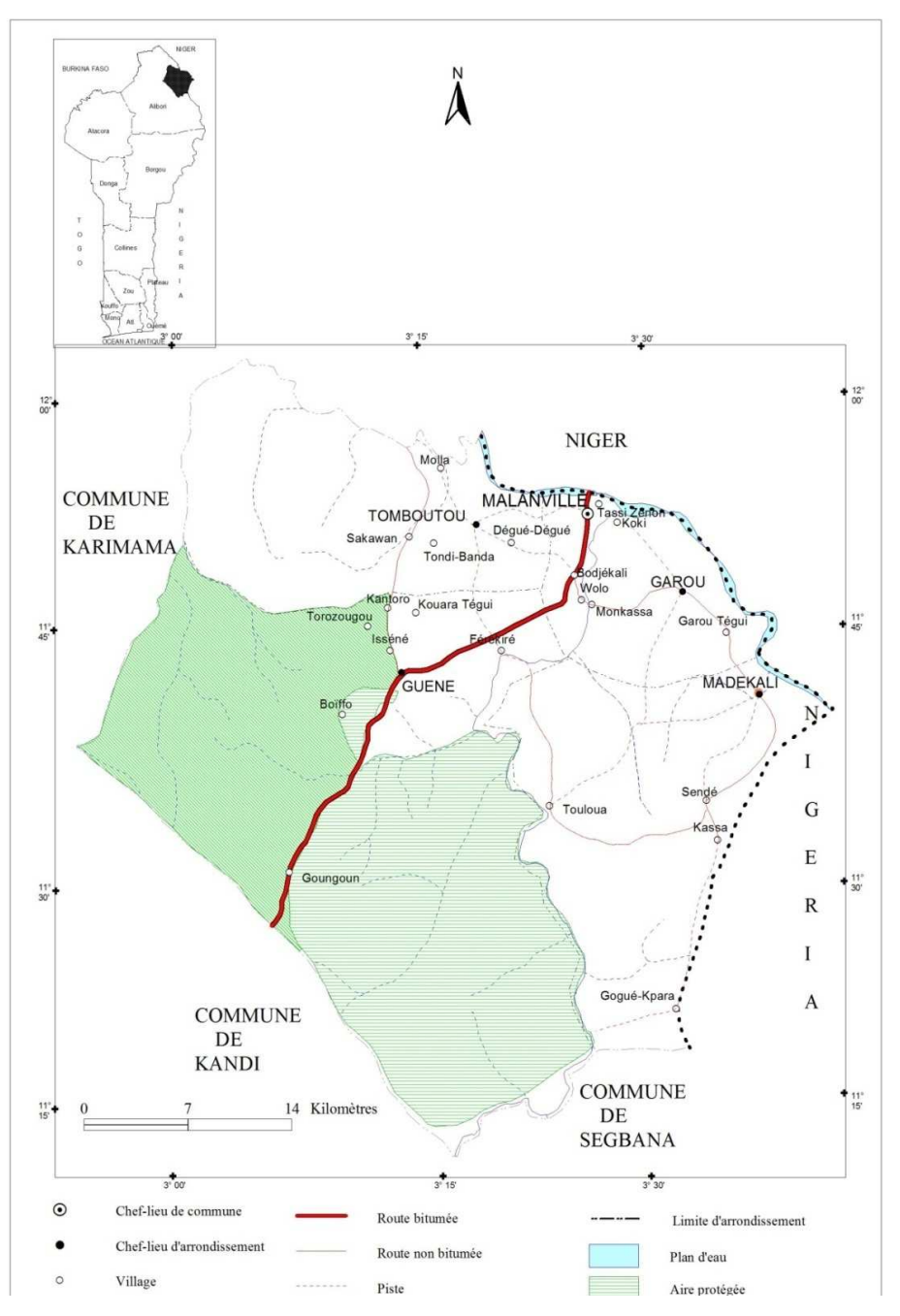

Figure 1: Situation géographique de la Commune de Malanville. 
La Commune de Malanville s'est développée dans la basse vallée du Niger autour du fleuve Niger. Elle occupe une bonne partie du lit majeur du fleuve Niger. Le Nord est une plaine alluviale et l'Est, une zone de dépression polymorphe traversée par la Sota, un affluent de la rive droite du Niger. A l'Ouest, se trouve un terrain sablonneux plus ou moins plat et drainé par l'Alibori, un autre affluent du Niger et au Sud, un terrain relativement plat. Dans les arrondissements de Madécali et Guéné, on rencontre des collines culminant à une hauteur moyenne de $80 \mathrm{~m}$. Ce relief est dans son ensemble développé dans une vallée alluvionnaire qui offre un cadre géomorphologique à la culture attelée.

\section{Approche méthodologique}

Afin de mener à bien le travail de terrain, des guides d'observation et d'entretien ont été élaborés. Des enquêtes socioéconomiques et agronomiques auprès des ménages agricoles permettent d'évaluer les aspects socio-économiques liés à la pratique de la culture attelée dans la Commune de Malanville.

Aussi, des observations directes relatives aux conditions de vie et d'entretien des animaux de trait, ainsi que le coût du matériel de culture attelée ont permis de faire un rapprochement entre les revenus et la culture attelée en milieu rural. Enfin, des informations ont été collectées auprès des institutions d'encadrement des paysans utilisateurs de charrue, de GV et des sages de la localité.

Les données d'enquête de terrain ont été obtenues auprès des paysans utilisateurs de charrues ainsi que des personnes ressources à partir du brainstorming. Ces dernières ont été dépouillées manuellement, ensuite introduites à l'ordinateur pour être traitées, puis transformées en tableaux et figures afin d'en faire des analyses et interprétations.

\section{Analyse statistique}

Les données ont été traitées à l'ordinateur avec le logiciel Excel. Certaines données ont été transformées en tableaux et d'autres en figures. La carte a été réalisée à l'aide du logiciel Arc view.

Les données d'enquête de terrain ont été obtenues auprès des paysans utilisateurs de charrues ainsi que des personnes ressources à partir du brainstorming. Ces dernières ont été dépouillées manuellement ensuite introduites à l'ordinateur pour être traitées.

Ainsi, des paramètres statistiques ont été calculés. Il s'agit: des paramètres de dispersion tels que les anomalies centrées qui ont été calculées par la formule $X-\bar{X}$ et les anomalies centrées réduites par la formule :

$$
\begin{array}{ll}
\frac{X-\bar{X}}{\sigma} \quad \text { Avec } & \frac{\mathrm{X}}{X}=\text { Variable } 1 \\
\sigma=\sqrt{\text { Variance }} &
\end{array}
$$

L'interprétation de ces anomalies a permis de mieux apprécier la tendance générale des prix de charrues qui est à la hausse de 1984 à 2008.

\section{RÉSULTATS}

Etat de la mécanisation agricole dans le Borgou et l'Alibori : introduction de la culture attelée comme un facteur favorable à l'émergence de la mécanisation

Les premières tentatives de l'introduction de la culture attelée ont eu lieu dans le Borgou et l'Alibori en 1930, dans la ferme expérimentale d'Ina au nord du Bénin. C'est le Projet Culture Attelée et Production de Viande dans les Départements du Borgou et de l'Alibori qui, dès 1965, a introduit la pratique de la culture attelée dans ces Départements dont la Commune de Malanville, comme méthode culturale.

Les expériences ont débuté par des essais de matériels tirés par les ânes. Ces expériences furent couronnées de succès, mais elles ont rencontré des difficultés du fait que les ânes posaient des problèmes sanitaires, du fait de leur sensibilité à la trypanosomiase d'une part, et de la non motivation des paysans d'autre part. Ce sont les raisons pour lesquelles, la vulgarisation de cette nouvelle technique a été très lente dans un premier temps, jusqu'au début des années 60 . 
$\mathrm{Au}$ lendemain de l'indépendance du Bénin, la culture attelée a été massivement expérimentée dans le Nord-Est du pays, avec le concours des organismes comme la Compagnie Internationale de Développement Rural (CIDR), l'Union Suisse des Coopératives (USC), la Food and Agriculture Organisation (FAO) et l'Association Française des Volontaires du Progrès (AFVP). Les actions conjuguées de ces différents organismes ont donné des résultats satisfaisants. C'est ainsi que naquit, le projet pour la relance de l'opération culture attelée dans le Nord du Bénin. Ce projet dirigé par la FAO, a démarré en 1965. Dès 1971, on notait :

- la mise en place de 1500 attelages avec les équipements correspondants ;

- la mise en place de 34 centres de dressage et d'un réseau d'ateliers artisanaux ;

- la promotion de groupements des usagers de la culture attelée.

Depuis lors, la culture attelée a commencé à prendre de l'ampleur dans le Nord du pays, et a gagné progressivement du terrain dans les autres régions du Bénin. Selon le Ministère de l'agriculture, il existe actuellement 92600 attelages sur le plan national dont $86 \%$ dans les Départements du Borgou et de l'Alibori.

\section{L'évolution de la production du coton}

Le coton est un produit stratégique pour l'économie béninoise. En effet, il procure un revenu à plus d'un tiers de la population. Mais aujourd'hui, cette culture enregistre des contreperformances imputables aux problèmes internes de gestion de la filière et aux subventions massives que les pays développés accordent à leurs producteurs. Si rien n'est fait, il va s'en suivre une aggravation sans précédent de la pauvreté des populations rurales, accompagnée de graves déficits budgétaires de l'économie béninoise.

L'analyse de la production cotonnière béninoise peut se faire sur deux périodes (Figures 2 et 3 ). Une première période allant de 1980 à 1999 caractérisée par une progression plus ou moins régulière de la production avec une tendance à la hausse. Une seconde période allant de 1999 à 2010 qui est caractérisée globalement par une tendance à la baisse avec une évolution erratique. On observe aussi sur cette deuxième période des efforts de relance caractérisés par des sursauts de reprise par endroits. Ainsi, pourrait-on évoquer les pics des campagnes 2001-2002 et 2007-2008 ainsi que le record de production de la campagne 2004-2005.

$\mathrm{Au}$ cours de la seconde période, la production moyenne a été d'environ 300000 tonnes par an pour une capacité d'égrenage dépassant 600000 tonnes. La production maximale enregistrée sur cette période a été de 426251 tonnes et est relative à la campagne 2004-2005. Elle n'a plus atteint 300 000 tonnes sur les cinq dernières campagnes qui ont suivie cet exploit malgré les efforts du gouvernement pour la relance de la production. Pour renverser la tendance, il est alors important, à partir des données empiriques, d'analyser les facteurs qui influencent la production du coton afin de proposer des stratégies complémentaires de relance de la sous filière coton-graine en complément de celles déjà proposées dans les OSD. Sur la base des données empiriques, un modèle économétrique a été construit pour analyser le lien fonctionnel qui existe entre l'offre du coton-graine et certaines variables d'intérêt. Compte tenu des données disponibles, une série chronologique de données sur la période de 1990 à 2009, soit sur 20 ans, a été utilisée.

\section{Contribution du coton à la formation du revenu agricole et l'emploi rural}

Le coton est la principale source de revenu monétaire des paysans qui le cultive. Il procure aux producteurs agricoles de la zone Nord du Bénin, environ deux-tiers à troisquarts de leurs revenus. La Figure 3 présente le revenu que le coton génère aux producteurs en termes de marge brute. 
Le coton génère un nombre très important d'emplois directs et indirects. En 2009, on dénombre environ 135635 cotonculteurs dont 25901 femmes. Selon le rapport conjoint de la FAO et de la BAD, en 2006 sur la filière coton en Afrique de l'Ouest, chaque tonne de coton-fibre représente de l'ordre de 320 hommes-jours de travail agricole assuré en partie par la main d'œuvre salariée. A cet emploi agricole s'ajoutent d'autre part les emplois salariés créés par les sociétés cotonnières estimés à 3500 emplois directs au plan national. Toutefois, ces statistiques non moins importantes, sont en baisse par rapport à leur niveau de 2004, liées à l'abandon de la filière par bon nombre de producteurs. Elles prouvent cependant, le potentiel de la filière coton à générer de l'emploi rural. Cette baisse des revenus a un impact direct sur le fonctionnement des producteurs et sur leur approvisionnement en outils de travail notamment l'attelage et les machines agricoles, dont le niveau de prix ne cesse d'augmenter.

\section{Les attelages et leur niveau de prix}

Le prix des attelages est en rapport avec les équipements agricoles qui les constituent. Il concerne la valeur de la paire de bœufs et celle des équipements.

\section{La paire de bœufs}

Achetée pour la plupart chez les éleveurs, la paire de bœufs a un prix qui varie selon les différentes régions du département de l'Alibori. Un prix moyen oscille autour de $80.000 \mathrm{~F}$ CFA pour la paire non dressée et 90.000 F CFA pour la paire dressée, soit un taux d'augmentation après dressage de 12,5\% par rapport au prix du marché d'achat.

\section{Lien entre production agricole et les prix des équipements agricoles}

Les revenus tirés du coton sont en grande partie investis dans l'achat des équipements. Les coûts du matériel agricole connaissent une augmentation régulière au fil des campagnes agricoles (Figure 2), contrairement à la production du coton. Cette augmentation est due à l'inflation que subissent les matières premières importées par la COBEMAG pour la fabrication des équipements agricoles.

La variabilité interannuelle des prix de charrues (Figure 2) de 1984 à 2008 montre que le prix augmente régulièrement chaque année. Par ailleurs, on constate également que cette augmentation n'est pas constante. Cette augmentation anarchique du coût de la charrue pose un problème en rapport aux moyens financiers du paysan. En calculant une anomalie centrée réduite, il ressort que le prix de la charrue augmente au fil des années, tendance qu'on peut ajuster à une fonction polynomiale $\mathrm{y}=0,18 \mathrm{x}-1,74$ avec un coefficient de régression $\mathrm{R}^{2}=0,96$ (Figure 3 ).

La tendance générale des prix de charrues est à la hausse de 1984 à 2008 (Figure 3). Cette situation ne permet pas à de nombreux paysans de pouvoir s'acheter leur propre charrue, surtout dans un contexte de pauvreté. Ceci constitue alors une contrainte à la culture attelée dans la Commune de Malanville.

\section{Durée d'utilisation et coût d'entretien des facteurs de production}

Les éléments d'appréciation de la durée d'utilisation et des coûts d'entretien des attelages sont des estimations faites auprès des agents de la section "culture attelée" de CeRPA Borgou et des paysans ayant une longue expérience en matière de culture attelée. Aucune précision exacte sur les périodes de renouvellement n'existe sur le matériel livré aux paysans. Il existe cependant quelques pièces détachées fréquemment remplacées par les paysans. La Coopérative Béninoise Matériel Agricole (COBEMAG) ne peut pas alors prévoir le nombre de pièces à produire pour satisfaire les besoins (Tableaux 1 et 2). 


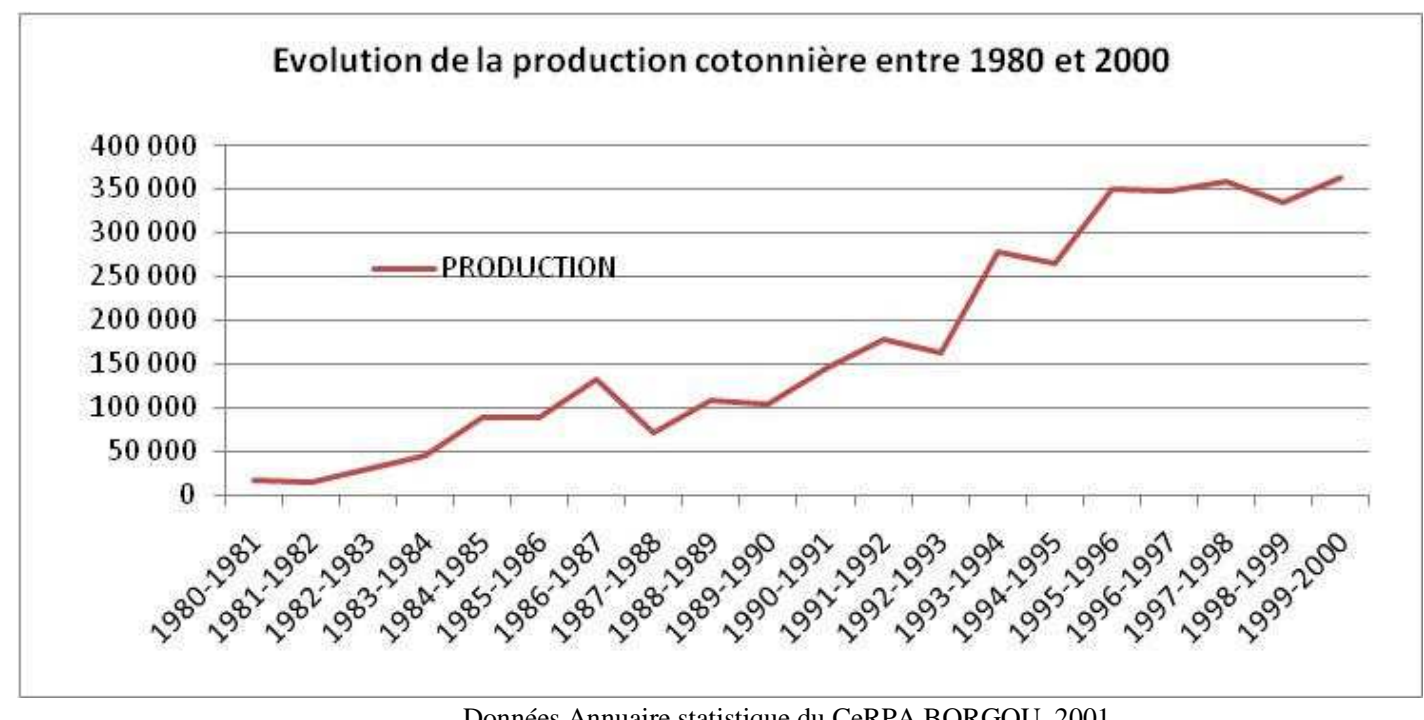

Données Annuaire statistique du CeRPA BORGOU, 2001.

Figure 2: Evolution de la production cotonnière au Bénin entre 1980 et 2000.

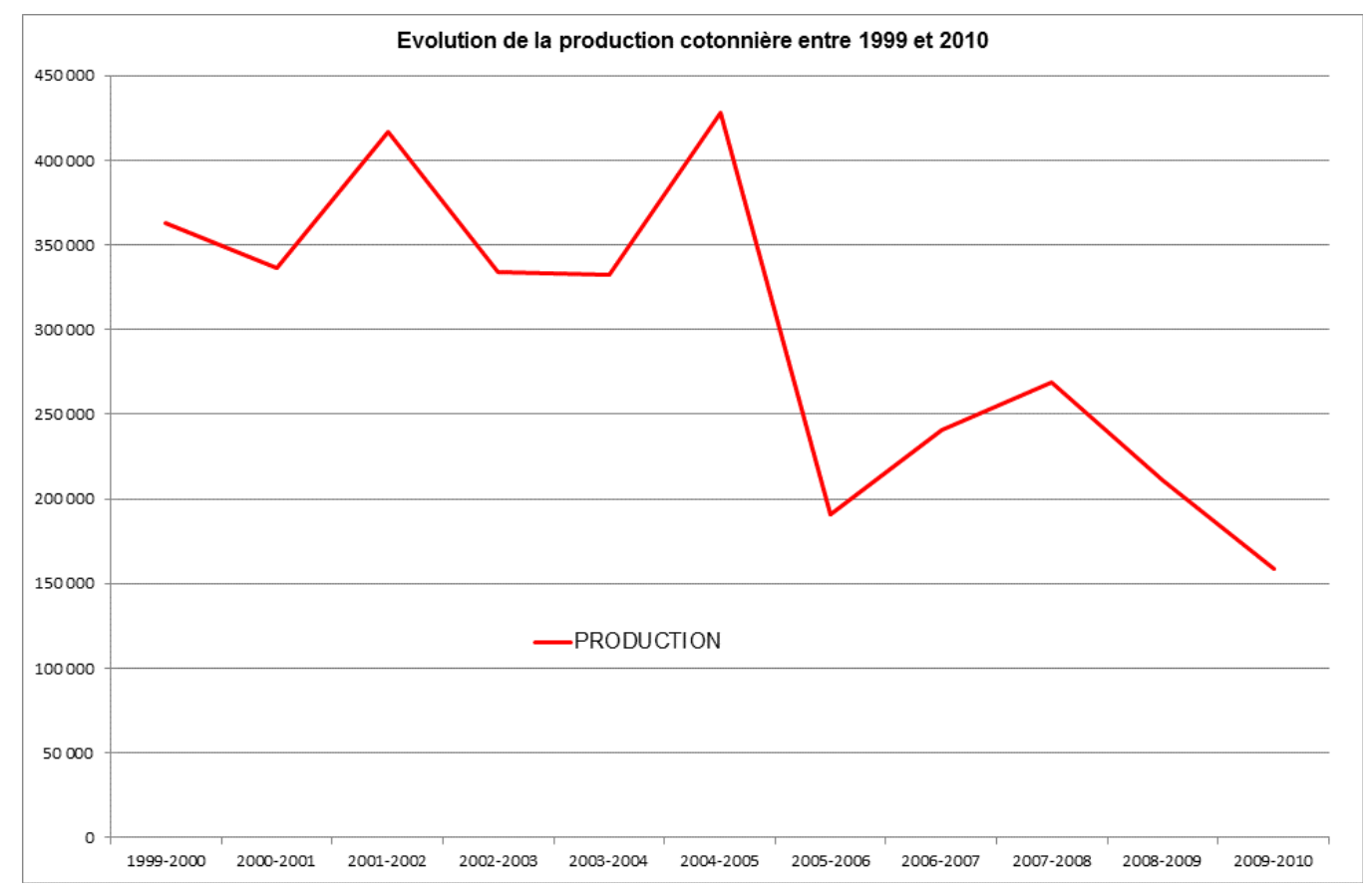

Figure 3: Evolution de la production cotonnière au Bénin entre 1999 et 2010. 


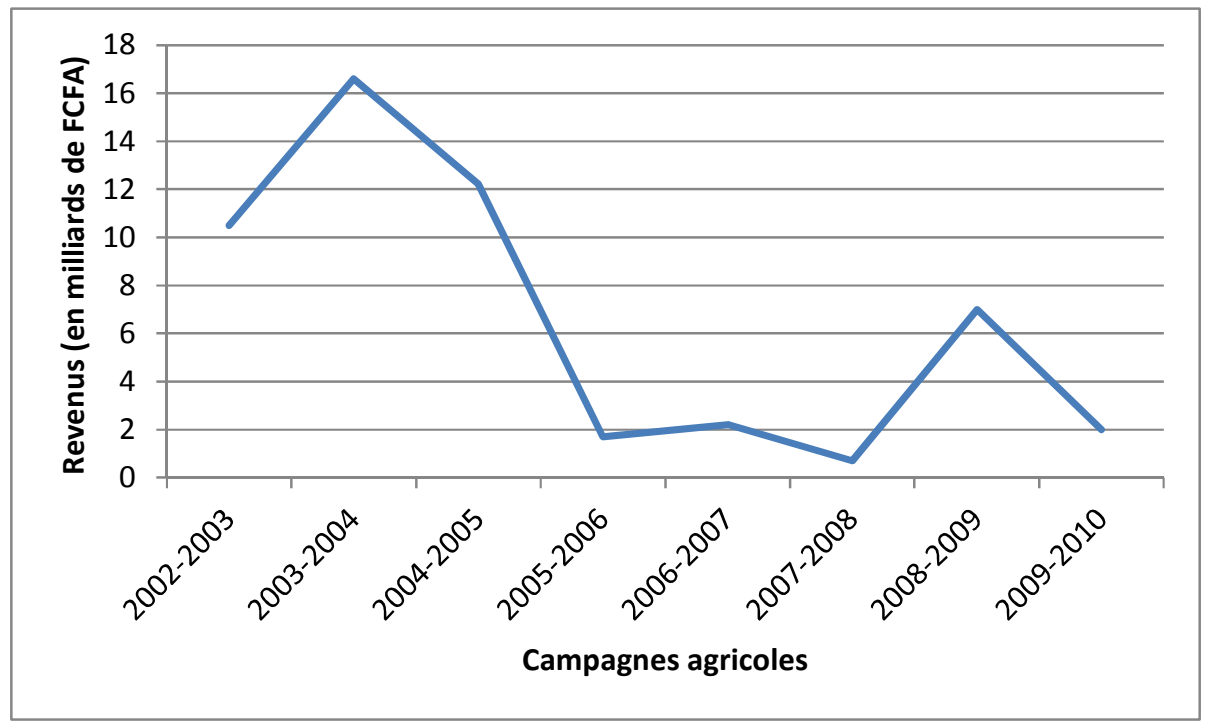

Figure 4: Evolution des producteurs de 2002 à 2010 au Bénin.

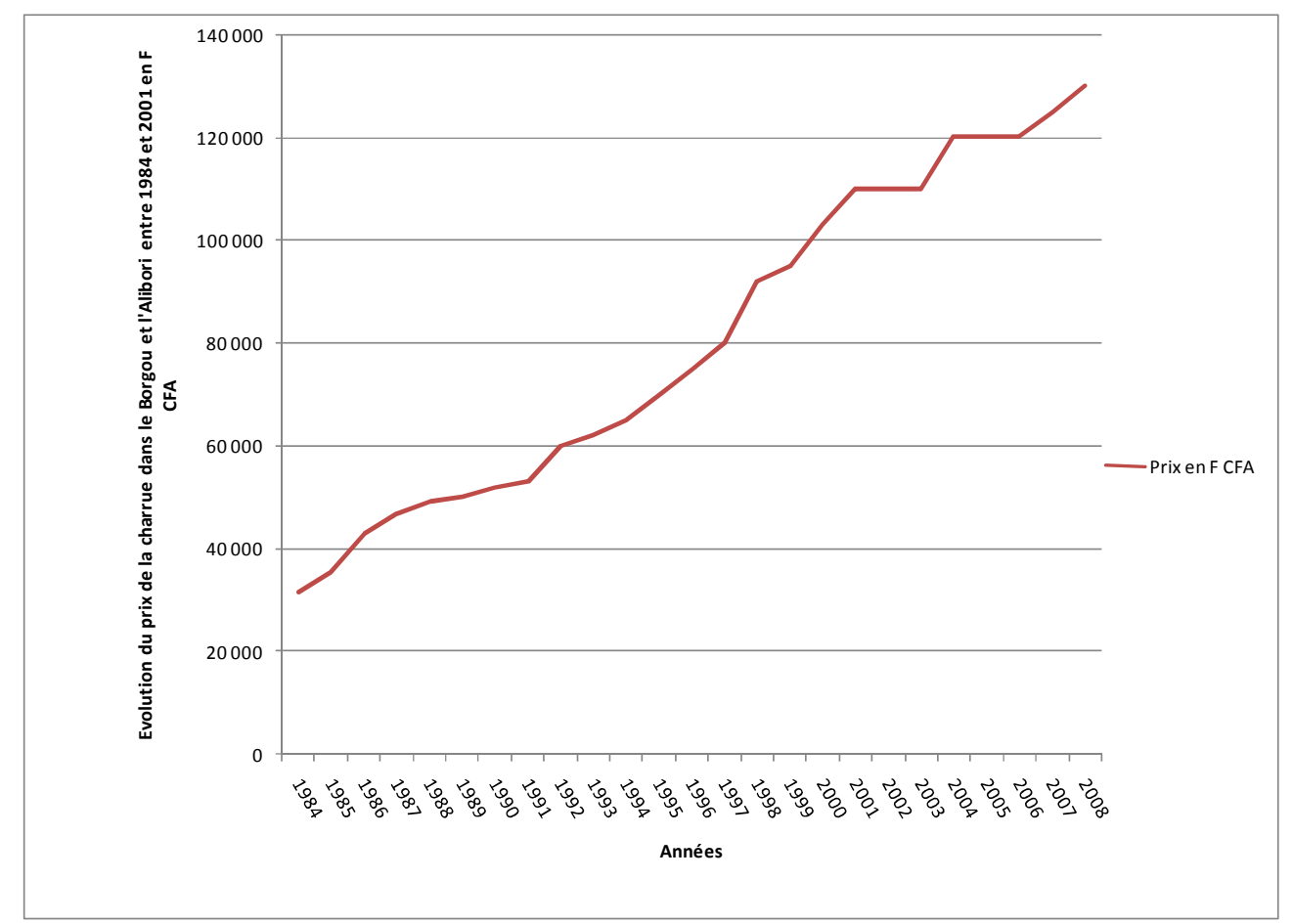

Figure 5 : Evolution des prix des charrues de 1984 à 2008. 


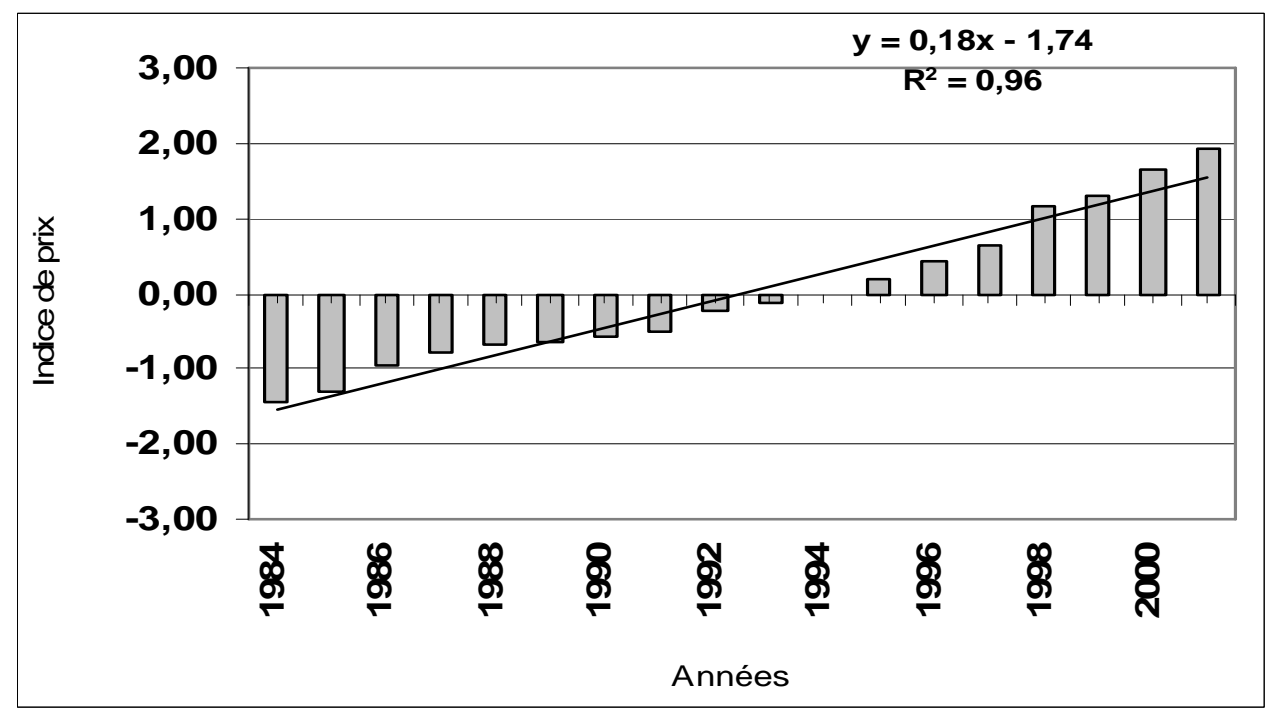

Source : CeRPA BORGOU ALIBORI ; 2008.

Figure 6: Variation du prix des charrues de 1984 à 2000 en termes d'anomalie centrée.

Tableau 1: Prix de cession et durée d'utilisation de la paire de bœufs de la charrue et des pièces usuelles acquises à crédit.

\begin{tabular}{lccc}
\hline Libellé & $\begin{array}{c}\text { Prix moyen de cession } \\
\text { (F.C.F.A.) }\end{array}$ & $\begin{array}{c}\text { Durée d'utilisation } \\
\text { (année) }\end{array}$ & $\begin{array}{c}\text { Amortissement } \\
\text { (F.C.F.A.) }\end{array}$ \\
\hline Paire de bœufs & 90.000 & 05 & - \\
Charrue complète & 47.775 & 10 & $4777,5(10 \%)$ \\
Pièces usuelles & 2500 & 0,5 & 5000 \\
soc & 1135 & 0,1 & 3560 \\
contre sept & 890 & 0,25 & 640 \\
talon & 1920 & 0,3 & 570 \\
roue & 570 & 0,1 & 112 \\
axe de roue & 560 & 0,5 & 105 \\
& & & 11017 \\
\hline \multicolumn{1}{c}{ Source: CeRPA BORGOU, 2008 } \\
\end{tabular}


Tableau 2 : Estimation du coût d'entretien annuel d'un attelage de paysan moyen pratiquant la culture attelée.

\begin{tabular}{lr}
\hline Animaux de trait & \\
Prix d'achat de la paire (FCFA) & 80.000 \\
Durée d'utilisation & $5 \mathrm{ans}$ \\
\hline Coût d'entretien/an (FCFA) & 15.000 \\
- Alimentation complémentaire (Pierre à lécher) & 3.000 \\
- Soins vétérinaires & 2.000 \\
- Charges annexes & 20.000 \\
Coût d'entretien/ jour (FCFA) : 20 000 : 365 = 54,79 & \\
Charrue et ses pièces & 47.775 \\
- Prix d'achat (FCFA) & $10 \mathrm{ans}$ \\
- Durée d'utilisation & \\
Coût d'entretien/an (FCFA) & 11.017 \\
- Maintenance & 30,18 \\
- Coût d'entretien/jour (FCFA) 11.017 : 365 \\
Coût total d'entretien de l'attelage / an (FCFA): \\
20. 000 + 11.017 \\
Coût total d'entretien de l'attelage /jour (FCFA) : \\
31.017 365 \\
\hline
\end{tabular}

Source: CeRPA BORGOU, 2008.

Les prix moyens de cession pour la maintenance des différentes pièces d'une charrue complète varient en fonction de l'importance de la pièce. Ainsi, pour la maintenance de la charrue, les paysans devront disposer d'un minimum de 11.017 FCFA.

En prenant en compte le coût de maintenance et avec une estimation du coût des différents matériels utilisés en culture attelée, nous avons évalué le coût d'entretien annuel et même journalier de l'attelage du paysan moyen pratiquant la culture attelée qui s'évalue à 31.017 F.CFA/an, soit 84,97 FCFA/jour (Tableau 2).

De l'analyse de ces tableaux, la culture attelée exige des investissements financiers afin d'assurer une utilisation efficiente de l'attelage. Il faut souligner que la majorité des paysans n'arrivent pas à effectuer tous ces investissements, faute de moyens malgré les nombreux avantages socio-économiques liés à la culture attelée.

Cependant, certains facteurs constituent un handicap à cette technique.
Les facteurs limitants de la culture attelée dans la zone d'étude

Les facteurs limitants de la culture attelée sont multiples. Ils se situent au niveau du paysan, de l'encadrement technique, de l'approvisionnement en matériel agricole, du suivi des animaux.

\section{Au niveau des paysans}

Ces facteurs se résument à la non maîtrise ou à la mauvaise utilisation des attelages pour les travaux agricoles. Cependant, les variables économiques telles que le revenu de la culture cotonnière, le nombre d'actifs familiaux de l'exploitation agricole, la superficie cultivée en coton et le capital d'exploitation; les variables sociales telles que l'âge et l'expérience du producteur dans la culture cotonnière; et la variable institutionnelle comme l'accès au crédit ont un impact positif sur la probabilité de choix de l'investissement en culture attelée au seuil de $5 \%$. 


\section{Au niveau de l'encadrement technique}

Il s'agit de l'insuffisance de l'encadrement technique dans certains arrondissements. La contrainte fondamentale est le manque de personnel compétent en la matière. L'encadrement technique est négligé par les agents eux-mêmes. Ces derniers préfèrent se livrer à d'autres activités à but lucratif comme le commerce. Toutefois, ceci ne constitue pas une reconversion socioprofessionnelle dans ce contexte de pauvreté dans le monde rural du septentrion.

\section{Au niveau de l'approvisionnement en matière agricole}

La Coopérative Béninoise de Matériel Agricole (COBEMAG) est la seule unité de fabrication de matériels agricoles. Les paysans de la Commune de Malanville se trouvent ainsi confronté au problème de ravitaillement. Ceci entraîne une sous utilisation de l'attelage, un rendement bas et un endettement excessif du paysan.

\section{DISCUSSION}

Les programmes visant à développer la culture attelée se heurtent à d'énormes difficultés. (Amadou, 1995, cité par Gnanro, 1996; CIRAD, 1996). Entre le début des années 70 et la fin des années 80, la diffusion et le financement de la traction animale, le choix des matériels agricoles, leur distribution, la formation des artisans et des paysans ainsi que les actions de vulgarisation technique étaient sous le contrôle des sociétés de développement agricole liées au coton ou à l'arachide.

Dans la Commune de Malanville, les éléments permettant l'adoption de la culture attelée sont nombreux : forte zone d'élevage, disponibilité de main d'œuvre. Mais, ce sont des éléments indispensables mais pas suffisants pour l'adoption de la culture attelée. En effet, dans le delta et une partie de la moyenne vallée du fleuve Sénégal, la traction animale n'a pas pu se développer pour les opérations culturales car les terres sont très argileuses et l'aridité du climat rend difficile l'alimentation des animaux de trait hors des casiers rizicoles (Beauval et Boquien, 2009).

Dans cette zone où la traction animale est dominante et dont le prix du matériel ne cesse de croître, le gros problème reste la quasi absence des crédits agricoles, éléments qui handicapent le développement de l'attelage malgré son efficience économique (Faure, 1994) et sa durabilité environnementale (Gibigaye, 2008; Tom et Wankpo, 2004). Malgré les incitations à l'utilisation de la traction animale, l'équipement peut être coûteux en situation de chute de la culture du coton. L'ensemble des équipements peut être onéreux par rapport au revenu actuel des ménages ruraux très pauvres de la Commune de Malanville. En 2008, une paire de bœufs plus la charrue et ses accessoires coûtaient environ 260 \$ pour l'ensemble. La seule paire de bœufs coûte 140 $\$$, par rapport au revenu annuel agricole du coton de $300 \$$ pour un producteur moyen, est une situation difficile pour pouvoir s'équiper en matériel. Et en absence de crédit destiné à l'achat des équipements, les producteurs doivent compter sur d'autres revenus notamment non agricoles qui parfois sont des éléments limitants dans le choix de la technologie. Comme facteurs limitants, il y a plusieurs niveaux d'analyse :

$\mathrm{Au}$ niveau des paysans selon MbétidBessane (2010), l'analyse économétrique des déterminants du choix de l'investissement en culture attelée à l'aide du modèle logit a révélé que la variable sociale telle que le niveau d'alphabétisation du producteur de coton n'a pas d'impact significatif sur la probabilité de choix. Ces résultats sont conformes aux travaux antérieurs sur les déterminants du choix des agriculteurs (Boussard, 1987 ; Mbétid-Bessane, 2003 ; Napier et al., 1991).

$\mathrm{Au}$ niveau de l'encadrement, selon Roesch (2004), dans les zones où la traction animale est peu présente, bon nombre d'exploitants hésitent à se lancer dans l'aventure. Ceux qui l'osent ont tous acquis une expérience, soit dans un centre de formation (opérations menées par les projets 
et $\mathrm{ONG}$ ), soit lors de migration temporaire dans des zones à forte implantation de traction animale où ils ont vu et pratiqué la culture attelée.

$\mathrm{Au}$ niveau de l'approvisionnement, selon Roesch (2004), la culture attelée se développe mieux et plus vite dans les zones où il existe déjà une « masse critique » d'attelages, là où il est possible pour des exploitants d'acheter du matériel d'occasion « pour se faire la main », là où peut jouer l'effet de formation de proximité et d'entraide. Parfois, la culture attelée est très anecdotique (Roesch, 2004) et réservée aux exploitations qui ont été en mesure de saisir une opportunité.

\section{Conclusion}

La culture attelée pratiquée dans le Borgou et l'Alibori a été introduite par l'intermédiaire de la culture de rente qui est le coton. Aujourd'hui, son usage est étendu aux autres cultures telles que le maïs, le sorgho, le mil, etc. Les nombreux avantages aussi bien sociaux qu'économiques que cette pratique offre aux paysans de la Commune de Malanville constituent une opportunité pour la promotion de cette technique culturale. C'est ce qui justifie le fait que malgré la cherté du matériel de culture attelée, les paysans, à défaut de pouvoir l'acheter individuellement se cotisent parfois de l'argent pour pouvoir se les acheter tantôt avec l'appui de la CLCAM à travers l'octroi de crédit aux paysans. C'est donc une technique qui a de l'avenir dans la Commune de Malanville. Il importe de donner assez de moyens aussi bien matériels que financiers à la commune afin que les paysans fassent de cette innovation un véritable instrument au service du développement.

Les analyses présentées confirment le rôle de la traction animale dans la conservation des sols. Toutefois, l'érosion et le ruissellement sont liés à la mauvaise formation des paysans et donc à une mauvaise utilisation du matériel agricole. Dès lors, le paquet technologique n'est pas bien assimilé par le monde paysan qui malgré tout, tient à la culture attelée.
Il ressort de toutes les analyses faites que la culture attelée présente un avantage en termes de rendement des cultures et de la facilité de travail. Elle constitue un atout pour l'agriculture dans la Commune de Malanville dans la vallée du Niger au Bénin. Elle permet la mise en valeur du milieu alluvionnaire. Cependant, son usage exige des investissements financiers qui constituent parfois des contraintes de la pratique de cette technique dans un contexte de pauvreté.

\section{REFERENCES}

Barro A, Zougmoré R, TaondaJ-B. S.2005. Mécanisation de la technique du zaï manuel en zone semi-aride. Cahiers Agricultures, 14(6): 549-559.

Beauval V, Boquien N. 2009. Mécanisation dans le contexte africain : notions préliminaires sur les techniques et enjeux. Grain de sel, 48: 13-14.

Boussard JM. 1987. Economie de l'agriculture. Economica, Paris, 310 p. de la culture attelée dans le centre-nord du Burkina Faso. Annales de l'Université de Ouagadougou, Série B, 001: 103-137.

Charrière G. 1984. La culture attelée: un progrès dangereux. Cah. ORSTOM, sér. Sci. Hum., 20(3-4): 647-656.

Gibigaye M, Sinsin B, Adégbidi A. 2006. Partage du risque en milieu rural au Bénin: cas des CUMA dans le Borgou. In Sciences de l'Environnement, Revue du Laboratoire de Recherches Biogéographiques et d'Etudes Environnementales (LaRBE). Presse de l'UL: Togo; 133-145.

Gibigaye M. 2008. La diffusion des innovations agricole dans le Borgou et l'Aliboriau Bénin. Cas des Coopératives d'Utilisation de Matériel Agricole (CUMA). Thèse de Doctorat Unique de l'Université d'Abomey-Calavi, EDP/ FLASH, Bénin, 243p.

Gnanro YS. 1996. Système agraire et dégradation du milieu physique dans la Sous-Préfecture de Gogounou. Mémoire de Maîtrise de Géographie, FLASH/UNB, 88p. 
Havard M, Lhoste P. 2009. Évolution de la traction animale en Afrique de l'Ouest et en Afrique Centrale. Grain de sel, 48: 1518.

Le Thiec G. 1996. Agriculture Africaine et Traction Animale. Cirad: Montpellier; $355 \mathrm{p}$.

Lhoste P. 1995. La Traction Animale en Afrique: Situation et Perspectives. FAO/CIHEAM: Saragosse; $14 \mathrm{p}$.

Mbétid-Bessane E. 2010. Investissement en culture attelée et amélioration de la rentabilité de la culture cotonnière en Centrafrique. Tropicultura, 28(3): 178183.

Mbétid-Bessane E. 2003. Crise cotonnière en Centrafrique et choix des agriculteurs en fonction de leur localisation à la ville. Tropicultura, 21(4): 218-220.

Napier TL, Napier AS, Turcker MA. 1991. The social, economic and institutional factors affecting adoption of soil conservation practices: the asian experience. Soil Tillage Research, 20: 365-382.
Pingali P, Bigot Y, Binswanger H.P. 1987. La Mécanisation Agricole et l'Evolution des Systèmes Agraires en Afrique Subsaharienne. Banque Mondiale: Washington; 206.

Roesch M. 2004. Financement de la culture attelée et stratégies d'équipement. Revue Élev. Méd. Vét. Pays Trop., 57(3-4): 191199.

Roupsard M. 1984. Le point sur la culture attelée et la mécanisation au NordCameroun. Cah. ORSTOM sér. Sci. Hum., 20(3-4): 613-631.

Tom P, Wankpo E. 2004. La production du Coton au Bénin: Résumé. Agriterra: Amsterdam, Pays bas; 3 .

Vall E, Lhoste P, Abakar O, Dongmo NA. 2003. La traction animale dans le contexte en mutation de l'Afrique subsaharienne: enjeux de développement et de recherche. Cahiers Agricultures, 12(4): 219-226. 